\title{
Towards a Room-Temperature Spin Quantum Bus in Diamond via Electron Photoionization, Transport, and Capture
}

\author{
M. W. Doherty, ${ }^{1, *}$ C. A. Meriles, ${ }^{2}$ A. Alkauskas, ${ }^{3}$ H. Fedder, ${ }^{4}$ M. J. Sellars, ${ }^{1,5}$ and N. B. Manson ${ }^{1}$ \\ ${ }^{1}$ Laser Physics Centre, Research School of Physics and Engineering, Australian National University, \\ Australian Capital Territory 2601, Australia \\ ${ }^{2}$ Department of Physics, CUNY-City College of New York, New York, New York 10031, USA \\ ${ }^{3}$ Center for Physical Sciences and Technology, Vilnius LT-10257, Lithuania \\ ${ }^{4}$ 3. Physikalisches Institut and Research Center SCOPE, \\ University Stuttgart, Pfaffenwaldring 57, 70569 Stuttgart, Germany \\ ${ }^{5}$ ARC Centre for Quantum Computation and Communication Technology, Australian National University, \\ Australian Capital Territory 2601, Australia
}

(Received 27 November 2015; revised manuscript received 23 September 2016; published 18 November 2016)

\begin{abstract}
Diamond is a proven solid-state platform for spin-based quantum technology. The nitrogen-vacancy center in diamond has been used to realize small-scale quantum information processing and quantum sensing under ambient conditions. A major barrier in the development of large-scale quantum information processing in diamond is the connection of nitrogen-vacancy spin registers by a quantum bus at room temperature. Given that diamond is expected to be an ideal spin transport material, the coherent transport of spin directly between the spin registers offers a potential solution. Yet, there has been no demonstration of spin transport in diamond due to difficulties in achieving spin injection and detection via conventional methods. Here, we exploit detailed knowledge of the paramagnetic defects in diamond to identify novel mechanisms to photoionize, transport, and capture spin-polarized electrons in diamond at room temperature. Having identified these mechanisms, we explore how they may be combined to realize an on-chip spin quantum bus.
\end{abstract}

DOI: 10.1103/PhysRevX.6.041035

\section{INTRODUCTION}

The success of quantum information processing (QIP) in diamond is owed to the remarkable nitrogen-vacancy (NV) center [1]. The spins of individual NV centers are optically addressable, such that light can be used to initialize and readout their electronic spin states [1]. Additionally, the NV center has the longest spin coherence time $\left(T_{2} \sim 2 \mathrm{~ms}\right)$ of any solid-state system at room temperature [2]. Using these properties, the room-temperature operation of spin registers has been demonstrated in diamond by constructing clusters of NV centers or combining NV centers with other paramagnetic defects, such as ${ }^{13} \mathrm{C}$ isotopic impurities and substitutional nitrogen $\mathrm{N}_{S}$ donor centers [3-10]. As the clusters are ultimately limited to a few spins, large-scale QIP in diamond requires many clusters to be networked together by quantum buses. To be scalable, the quantum buses must be sufficiently compact to be incorporated on chip [11]. Furthermore, to exploit diamond's primary advantage over competing QIP platforms, such as superconducting qubits

*marcus.doherty@anu.edu.au

Published by the American Physical Society under the terms of the Creative Commons Attribution 3.0 License. Further distribution of this work must maintain attribution to the author(s) and the published article's title, journal citation, and DOI.
Subject Areas: Quantum Information,

Semiconductor Physics, Spintronics
[12-14] and phosphorus dopants in silicon [15-17], the quantum buses should ideally be capable of roomtemperature operation. Beyond QIP, such a spin quantum bus will enable quantum-enhanced sensitivity of diamond's diverse nanometrology applications $[18,19]$.

At cryogenic temperatures, the coherence of the center's optical transitions has been exploited to entangle two spin registers separated by macroscopic distances via an optical quantum bus [20-22]. However, since the coherence of the center's optical transitions is lost above $\sim 35 \mathrm{~K}$, an optical quantum bus is not possible at room temperature [23]. An alternative currently being explored is to use phonons of nanomechanical oscillators, rather than optical photons, to mediate entanglement between distant spins [24-30]. But this alternative is also limited to cryogenic temperatures.

One suggestion for the realization of a quantum bus at room temperature has been to extend a chain of defect spins between two spin registers [31,32], but this is difficult to engineer and requires significant resources to control. An attractive alternative is the coherent electrical transport of a spin from one spin register to the other. Diamond presents as an ideal spin transport material: its large electronic band gap, inversion symmetry, small spin-orbit interaction, and low nuclear spin density promise long spin-relaxation times [33]. Indeed, $a b$ initio calculations predict a transport $T_{1} \sim 180 \mathrm{~ns}$ at room temperature (10 times longer than 
in silicon) [33], which corresponds to an exceptional transport distance of $\sim 2 \mathrm{~mm}$ in high-purity diamond [34] with a modest electric field of $\sim 100 \mathrm{~V} / \mathrm{cm}$. Since only paramagnetic impurities (whose densities can be controlled) and magnetic field inhomogeniety offer additional dephasing mechanisms, transport is also expected to be coherent, with ultimately $T_{2} \sim T_{1}$ at room temperature $[2,36]$. Since coherent electrical spin transport can be controlled by nanoelectrodes [37], which can be arranged in a scalable architecture [38], it poses as a suitable quantum bus for scalable diamond QIP devices that can operate at room temperature. This is a possibility that appears to be unique to diamond due to its extreme properties and unique defects.

Despite this ample motivation, virtually no spin transport experiments in diamond have been reported. This palpable deficiency has been due to the apparent absence of any mechanism to inject spin into diamond [39]. Similar to silicon [40], the indirect band gap of diamond precludes conventional optical orientation via circularly polarized light and conventional electrical injection is expected to be prevented by difficulties in interfacing ferromagnetic electrodes with diamond. While advances in the interfacing of ferromagnetic electrodes ultimately enabled spin injection in silicon [40], this method is not suitable for coherent transport between spin registers, and neither is the recent proposal to spin polarize carriers in conductive dopant wires in diamond heterostructures via Overhauser cross relaxation with optically pumped ensembles of $\mathrm{NV}$ centers [41].

In this paper, we explore how spin-coherent photoionization, transport, and capture of electrons in diamond can be used to realize an on-chip spin quantum bus. We begin by reviewing the unique properties of the $\mathrm{NV}$ and $\mathrm{N}_{S}$ centers. In doing so, we identify how $\mathrm{NV}_{-}{ }^{14} \mathrm{~N}_{S}$ defect pairs can be used to achieve spin-coherent electron photoionization and capture. In subsequent sections, we combine the $\mathrm{NV}-{ }^{14} \mathrm{~N}_{S}$ mechanisms with a drift-diffusion model of electron transport to describe the coherent transport of spin between distant spin clusters. We find that clusters must be connected by diamond nanowires in order to sufficiently control the electron transport. Finally, we propose protocols for entangling distant spin clusters via spin-coherent electron transport and estimate that entanglement fidelities sufficient for fault-tolerant QIP can be achieved at cryogenic temperatures and, with further nanowire engineering, potentially at room temperature.

\section{NV AND N $_{S}$ CENTERS}

\section{A. NV center}

The NV center is a point defect composed of a substitutional nitrogen-carbon vacancy pair orientated along the $\langle 111\rangle$ crystallographic direction [see Fig. 1(a) for diagram and Ref. [1] for an extensive review of the NV center]. It is

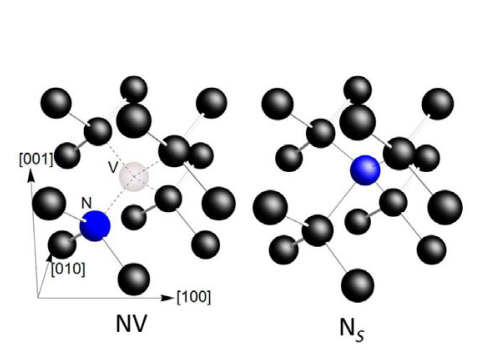

(a)

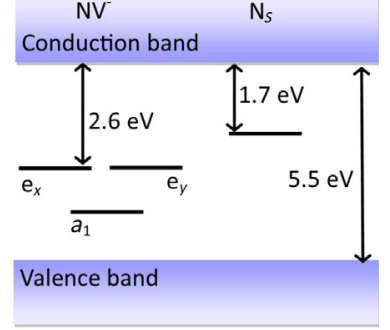

(b)

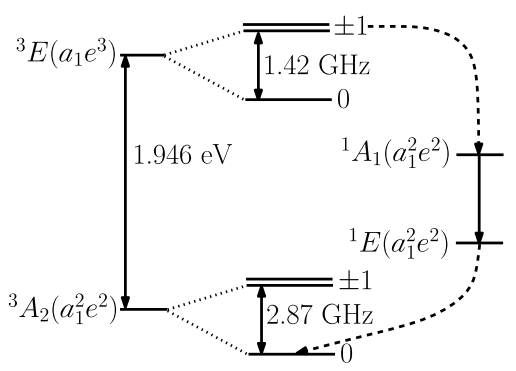

(c)

FIG. 1. (a) Diagrams of the $\mathrm{NV}$ and $\mathrm{N}_{S}$ centers that depict carbon (black) and nitrogen (blue) atoms and the vacancy (transparent). (b) Electronic band structure diagram depicting the defect levels of the $\mathrm{NV}\left(a_{1}, e_{x}, e_{y}\right)$ and $\mathrm{N}_{S}$ centers. The diamond indirect band gap and defect photoionization energies are as indicated $[42,43]$. (c) Electronic structure of the $\mathrm{NV}^{-}$center depicting the electronic levels (with corresponding electronic configurations in parentheses) and fine structure states (denoted by electronic spin projection) [1]. The visiblezero-phonon line energy and the zero-field fine-structure splittings of the triplet levels are as indicated. Solid arrows represent optical or spin transitions, whereas dashed arrows represent the spin-selective nonradiative ISCs that lead to optical spin polarization and readout.

stable in neutral $\left(\mathrm{NV}^{0}\right)$ and negative $\left(\mathrm{NV}^{-}\right)$charge states, which are characterized by their optical zero-phonon lines at $2.156 \mathrm{eV}\left(\mathrm{NV}^{0}\right)$ and $1.946 \mathrm{eV}\left(\mathrm{NV}^{-}\right) . \mathrm{NV}^{-}$possesses the required properties for quantum technology and so is the desired charge state in thermal equilibrium. The thermal charge state is determined by the local density of electron donors and acceptors. $\mathrm{N}_{S}$ is the most prevalent donor in diamond and naturally coexists with the NV center as a byproduct of NV formation: either through N CVD doping or ion implantation. Indeed, localized $\mathrm{NV}-\mathrm{N}_{S}$ clusters, in which the $\mathrm{N}_{S}$ preferentially donates its electron to the $\mathrm{NV}$ to form $\mathrm{NV}^{-}$, can be created by ion implantation $[3,44]$.

The observable electronic structures of $\mathrm{NV}^{0}$ and $\mathrm{NV}^{-}$ are defined by the occupation of the center's three deep-level defect orbitals $\left(a_{1}, e_{x}, e_{y}\right)$ by three and four electrons, respectively [see Fig. 1(b)]. The electronic structure of $\mathrm{NV}^{-}$is depicted in Fig. 1(c) and consists of ground and optically excited spin triplet levels $\left({ }^{3} A_{2}\right.$ and $\left.{ }^{3} E\right)$ as well as intermediate spin singlet levels $\left({ }^{1} E\right.$ and $\left.{ }^{1} A_{1}\right)$. At room temperature, the observable fine structures of the ${ }^{3} A_{2}$ and ${ }^{3} E$ levels are analogous and can be described by the spin Hamiltonian 


$$
H_{\mathrm{NV}}=D\left(S_{z}^{2}-\frac{2}{3}\right)+\gamma_{e} S_{z} B
$$

where $D$ is the zero-field splitting arising from spin-spin interaction that is equal to $2.87 \mathrm{GHz}$ for ${ }^{3} A_{2}$ and $1.42 \mathrm{GHz}$ for ${ }^{3} E, S_{z}$ is an $S=1$ dimensionless electron spin operator, $\gamma_{e}=g_{e} \mu_{B} / h, \mu_{B}$ is the Bohr magneton, $g_{e} \sim 2$ is the $\mathrm{NV}^{-}$ electron $g$ factor that is approximately the isotropic freeelectron value, $h$ is the Planck constant, and $B$ is the applied magnetic field that is aligned with the center's $\langle 111\rangle$ trigonal symmetry axis. Note that hyperfine interactions with the intrinsic $\mathrm{N}$ nucleus and interactions with electric and strain fields are typically of the order of $\sim 1 \mathrm{MHz}$ and are not included in Eq. (1) because they do not significantly influence the discussion we present here.

Optical spin polarization and readout of $\mathrm{NV}^{-}$is enabled by spin-dependent nonradiative intersystem crossings (ISCs) between the spin triplet and singlet levels [depicted in Fig. 1(c)] [45,46]. Upon optical excitation, the ISCs offer an additional decay pathway from ${ }^{3} E$ to ${ }^{3} A_{2}$ that preferentially depopulates $m_{s}= \pm 1$ and populates $m_{s}=0$. After a few optical cycles the spin is polarized into $m_{s}=0$ with high fidelity. The additional nonradiative decay pathway is also responsible for the contrast in the center's fluorescence intensity between $m_{s}=0$ and \pm 1 . This contrast is used to optically readout the relative probability that the $\mathrm{NV}^{-}$spin is in the $m_{s}=0$ state. The time required to polarize or readout the spin is defined by the lifetime $\sim 300 \mathrm{~ns}$ of the ${ }^{1} E$ intermediate singlet level [1]. With the addition of an appropriately tuned magnetic field, the $\mathrm{NV}^{-}$electron spin can also be used for high-fidelity projective initialization and readout of coupled nuclear spins $[10,47,48]$.

As we describe in detail in Appendix A, the NV center may be photoconverted between $\mathrm{NV}^{-}$and $\mathrm{NV}^{0}$ [42]. Analysis of the one- and two-photon photoionization processes of $\mathrm{NV}^{-}$reveals that they do not maintain a high level of spin coherence for the ionized electron (see Appendix A). Thus, it is necessary to turn to the $\mathrm{N}_{S}$ center to achieve photoionization with high spin coherence. Note that another donor center, the substitutional phosphorus $\mathrm{P}_{S}$ center, is considered in Appendix B, but is found unsuitable.

\section{B. $\mathbf{N}_{S}$ center}

The $\mathrm{N}_{S}$ center is a deep donor in diamond and is known to exist in neutral $\left(\mathrm{N}_{S}^{0}\right)$ and positive $\left(\mathrm{N}_{S}^{+}\right)$charge states. In $\mathrm{N}_{S}^{0}$, the $\mathrm{N}_{S}$ donor level is occupied by one electron, whereas in $\mathrm{N}_{S}^{+}$it is unoccupied. There is disagreement about the energy of the $\mathrm{N}_{S}$ donor level below the conduction band, with various photoabsorption and photoconductivity measurements reporting the energy in the broad range $1.7-2.2 \mathrm{eV}$ [43,49-51]. This disagreement is likely due to a weak onset of photoconduction, thereby making the measurement of the onset energy susceptible to detection sensitivity. Consequently, it is likely that the donor level exists towards the lower bound of the range, near $1.7 \mathrm{eV}$, as supported by ab initio calculations [52].
We thus conclude that $\mathrm{N}_{S}^{0}$ will photoionize via the absorption of one photon with energy $>1.7 \mathrm{eV}$ and the ejection of an electron into the conduction band. Since photons with energy $>1.946 \mathrm{eV}$ are required to optically excite or photoionize $\mathrm{NV}^{-}$, it follows that a $\mathrm{N}_{S}^{0}$ center may be selectively photoionized using photons with energy < $1.946 \mathrm{eV}$ without affecting proximal $\mathrm{NV}^{-}$. Once ionized, the $\mathrm{N}_{S}^{+}$may be converted back to $\mathrm{N}_{S}^{0}$ by capturing an electron either from the conduction band via multiphonon emission or from the valence band via absorption of a UV photon with energy $>3.8 \mathrm{eV}$. Ignoring the latter, for a diffusing conduction electron density $\rho$ at the $\mathrm{N}_{S}$ center, the capture rate is $\Gamma_{\text {cap }}=\rho \sigma_{\text {cap }} \sqrt{k_{B} T / m}$, where $\sigma_{\text {cap }} \sim$ 3-7 $\mathrm{nm}^{2}$ [53] is the capture cross section of $\mathrm{N}_{S}^{0}, k_{B}$ is Boltzmann's constant, $T$ is temperature, and $m$ is the effective electron mass [54].

In greater detail, photoionization and capture involve an electron transiting between a $\mathrm{N}_{S}$ defect spin orbital and a conduction band Bloch spin orbital $\phi_{n, \vec{k}}$, where $n$ denotes the band and $\vec{k}$ is the Bloch wave vector. Considering just photoionization near threshold (i.e., photon energy between 1.7 and $1.946 \mathrm{eV}$ ) and capture of conduction electrons in thermal equilibrium, photoionization and capture will involve only Bloch spin orbitals that belong to the lowest conduction band and have wave vectors near the conduction band minimum. Diamond is a valley conductor with a conduction band minimum at the $\Delta \sim 0.77 X k$ point [35,55]. Near the minimum, the lowest conduction band is nondegenerate and transforms as the $\Delta_{1}$ representation. This implies that there is no spin-orbit interaction near the conduction band minimum [56]. Hence, photoionization and capture are spin conserving because both the $\mathrm{N}_{S}$ defect and Bloch spin orbitals have the same well-defined spin.

Considering now the fine details of the $\mathrm{N}_{S}$ center, from electron spin resonance (ESR) studies, it is known that $\mathrm{N}_{S}^{0}$ is distorted from the $T_{d}$ symmetry of the simple substitutional site to $C_{3 v}$ symmetry by the elongation of one of the four $\mathrm{N}-\mathrm{C}$ bonds, and is thus $\langle 111\rangle$ orientated [57]. As is often mistaken, this distortion is not a Jahn-Teller effect because the ground electronic state has $A$ symmetry in the undistorted configuration $[58,59]$. However, analogous to a JahnTeller distortion, $\mathrm{N}_{S}^{0}$ undergoes temperature-dependent reorientation between its four equivalent distortions [60]. At room temperature, the reorientation rate is $\sim 0.3 \mathrm{kHz}$. The $\mathrm{N}_{S}^{0}$ ESR does not exhibit zero-field fine structure from spinorbit interactions and its electron $g$ factor is approximately isotropic and equal to the free-electron value [57]. Including hyperfine interactions, the spin Hamiltonian of $\mathrm{N}_{S}^{0}$ is

$$
H_{\mathrm{N}}=\gamma_{e} s_{z} B+\vec{s} \cdot \mathbf{A} \cdot \vec{I}+\vec{I} \cdot \mathbf{Q} \cdot \vec{I}+\gamma_{n} I_{z} B
$$

where $\vec{s}$ are the $S=1 / 2$ dimensionless electron spin operators, $\vec{I}$ are the dimensionless nuclear spin operators, 
$\mathbf{A}$ and $\mathbf{Q}$ are the magnetic and electric hyperfine tensors, respectively, that depend upon the orientation of the center and the principal magnetic $\left(A_{\|}, A_{\perp}\right)$ and electric $(Q)$ hyperfine parameters, $\gamma_{n}=g_{n} \mu_{N} / h, g_{n}$ is the nuclear $g$ factor, and $\mu_{N}$ is the nuclear magneton. Note that the spin quantization axes in Eq. (2) are chosen to be parallel with the applied magnetic field $B$.

As can be seen in Table I, the ${ }^{15} \mathrm{~N}_{S}^{0}$ hyperfine parameters are of the magnitude $\sim 10^{2} \mathrm{MHz}$, which poses a major problem to spin-coherent photoionization and capture at centers with the ${ }^{15} \mathrm{~N}$ isotope. Owing to the stochastic nature of photoionization, the time the ${ }^{15} \mathrm{~N}_{S}^{0}$ ionizes is not precisely known. Because of the uncertainty in this ionization time, the ${ }^{15} \mathrm{~N}_{S}^{0}$ electron spin acquires an unknown phase through hyperfine interaction with the ${ }^{15} \mathrm{~N} I=1 / 2$ nuclear spin. The same is true when the electron is captured: the electron spin gains an unknown phase through hyperfine interactions at the new center because the capture time is not precisely known. Thus, these hyperfine interactions will dephase the electron spin on a time scale of $\sim 10 \mathrm{~ns}$ during photoionization and capture. This dephasing can be avoided only by engineering photoionization and capture to occur on much shorter time scales (i.e., «10 ns). However, this presents a major technical challenge, and on this basis, we rule out ${ }^{15} \mathrm{~N}_{S}$ as being a viable option for realizing a spin quantum bus. We note that ${ }^{15} \mathrm{~N}_{S}$ can, however, be used to simply produce incoherent spin-polarized conduction electrons.

The situation is different for ${ }^{14} \mathrm{~N}_{S}^{0}$ because the dephasing due to hyperfine interactions can be avoided by preparing the $I=1$ spin of ${ }^{14} \mathrm{~N}_{S}^{0}$ into the $m_{I}=0$ nuclear spin projection, which has no magnetic hyperfine interaction with the electron spin. Several techniques for this preparation have been devised and demonstrated $[3,4,8,9]$. Each involves optically spin polarizing a proximal $\mathrm{NV}^{-}$and transferring this polarization to the nuclear spin of the ${ }^{14} \mathrm{~N}_{S}^{0}$ center via the dipolar coupling of the $\mathrm{NV}^{-}$and ${ }^{14} \mathrm{~N}_{S}^{0}$ electron spins. Given this preparation, the electron spin dephasing rate is reduced to the rate at which the nuclear spin flips to $m_{I}= \pm 1$ and returns hyperfine interactions. The nuclear spin-flip rate is determined by the nonsecular terms in Eq. (2), which exist if the orientation of the magnetic field (aligned with the axis of the proximal $\mathrm{NV}^{-}$) differs from the orientation of the ${ }^{14} \mathrm{~N}_{S}^{0}$ center. Because of the continuous (slow) reorientation of the $\mathrm{N}_{S}^{0}$ center at room

TABLE I. Spin parameters of $\mathrm{N}_{S}^{0}$ taken from Ref. [59]: orientation of defect structure or principal hyperfine axis, electronic $g$ factor, nuclear spin of isotope, and magnetic $\left(A_{\|}, A_{\perp}\right)$ and electric $(Q)$ hyperfine parameters.

\begin{tabular}{lccccccc}
\hline \hline Defect & Orientation & $g_{e}$ & $g_{n}$ & $I$ & $A_{\|}(\mathrm{MHz})$ & $A_{\perp}(\mathrm{MHz})$ & $Q(\mathrm{MHz})$ \\
\hline${ }^{14} \mathrm{~N}_{S}^{0}$ & $\langle 111\rangle$ & 2 & +0.40 & 1 & 114 & 81 & -3.97 \\
${ }^{15} \mathrm{~N}_{S}^{0}$ & $\langle 111\rangle$ & 2 & -0.57 & $1 / 2$ & -160 & -114 & $\cdots$ \\
\hline \hline
\end{tabular}

temperature, differences in orientation are unavoidable. The effect of the nonsecular terms can be minimized by the application of a sufficiently large magnetic field (e.g., $B \sim 5000 \mathrm{G} \gg A_{\perp} / \gamma_{e} \sim 50 \mathrm{G}$ ), such that nonsecular terms between electron spin projections can be ignored, in which case, Eq. (2) becomes for ${ }^{14} \mathrm{~N}_{S}$

$$
\begin{aligned}
H_{\mathrm{N}} \approx & \gamma_{e} s_{z} B+\chi A_{\|} S_{z} I_{z^{\prime}}+Q\left[I_{z^{\prime}}^{2} \cos ^{2} \alpha+I_{x^{\prime}}^{2} \sin ^{2} \alpha-\frac{2}{3}\right] \\
& +\gamma_{n} B I_{z^{\prime}} \cos \alpha-\frac{Q}{2}\left(I_{x^{\prime}} I_{z^{\prime}}+I_{z^{\prime}} I_{x^{\prime}}\right) \sin 2 \alpha \\
& +\gamma_{n} B I_{x^{\prime}} \sin \alpha,
\end{aligned}
$$

where $\chi=1$ if the orientation of the ${ }^{14} \mathrm{~N}_{S}^{0}$ center is aligned with the magnetic field and $\chi \approx \sqrt{5} / 3$ if not, and the nuclear spin quantization axis has been rotated by an angle $\alpha$ from the magnetic field $B$, as denoted by the replacement $I_{x}, I_{y}, I_{z} \rightarrow I_{x^{\prime}}, I_{y^{\prime}}, I_{z^{\prime}}$. The angle $\alpha$ is defined by $\tan \alpha=0 \quad$ or $\quad \tan \alpha=-\left(A_{\|}-A_{\perp}\right) \sin 2 \theta /\left[A_{\|}+A_{\perp}+\right.$ $\left.\left(A_{\|}-A_{\perp}\right) \cos 2 \theta\right]$ for aligned or not aligned, respectively, where $\theta=\arccos (-1 / 3)$ is the tetrahedral angle. The final two terms drive the nuclear spin flips and are of the order of $\sim 1 \mathrm{MHz}$. A very conservative estimate of the nuclear spinflip rate is, thus, $\sim 1 \mathrm{MHz}$, although the actual rate is likely to be much smaller due to the $>80-\mathrm{MHz}$ secular magnetic hyperfine splitting of the nuclear spin states.

This conservative estimate of the nuclear spin-flip rate defines the maximum allowable period $(\sim 1 \mu \mathrm{s})$ between preparation of the ${ }^{14} \mathrm{~N}_{S}^{0}$ nuclear spin and photoionization or capture. During this period, two-qubit gates must be applied to the $\mathrm{NV}^{-}$and ${ }^{14} \mathrm{~N}_{S}^{0}$ electron spins in order to prepare or detect the ${ }^{14} \mathrm{~N}_{S}^{0}$ electron spin state. It is therefore an important remaining question as to whether these gates can be performed sufficiently fast. Their speed is limited by the dipolar coupling between the electron spins, which is a function of the distance between them. Since $\mathrm{NV}^{-}{ }_{-}^{14} \mathrm{~N}_{S}^{0}$ pairs with separations as small as $1.5 \mathrm{~nm}$ and dipolar couplings as large as $14 \mathrm{MHz}$ have been engineered by ion implantation in the past, it appears that this speed requirement can be met with adequate engineering. Hence, we conclude that strongly coupled $\mathrm{NV}^{-}{ }^{14} \mathrm{~N}_{S}$ pairs are suitable for spin-coherent photoionization and capture.

\section{SPIN-COHERENT PHOTOIONIZATION AND CAPTURE}

In this section, we apply the conclusions of the previous section to describe how an electron with a well-defined spin state $|\psi\rangle=\alpha|\uparrow\rangle+\beta|\downarrow\rangle$ can be photoionized from one $\mathrm{NV}-{ }^{14} \mathrm{~N}_{S}$ pair and subsequently captured and optically detected at another $\mathrm{NV}_{-}{ }^{14} \mathrm{~N}_{S}$ pair. We assume that the NV centers in the two clusters have the same orientation and the applied magnetic field is aligned with this orientation.

As depicted in Fig. 2(a), three steps are required to photoionize an electron with spin state $|\psi\rangle$ using a 


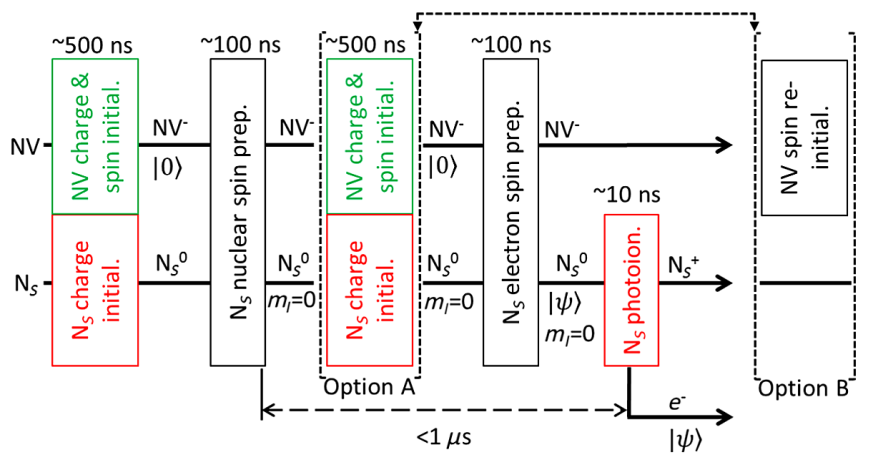

(a)

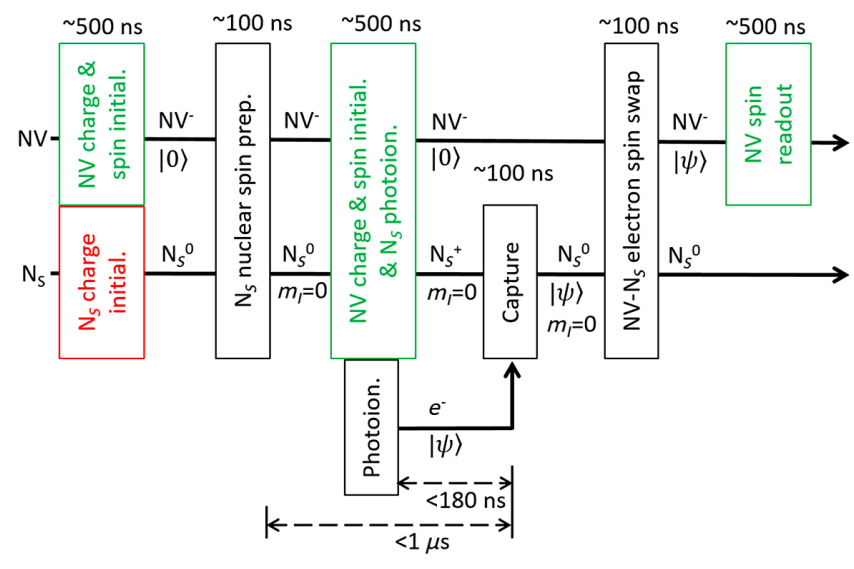

(b)

FIG. 2. Techniques to (a) photoionize an electron with spin state $|\psi\rangle$ from a NV- ${ }^{14} \mathrm{~N}_{S}$ pair and to (b) subsequently capture and optically detect its spin at another $\mathrm{NV}_{-}{ }^{14} \mathrm{~N}_{S}$ pair. Each box corresponds to an optical or microwave pulse described in the text and has its indicative length denoted above. For optical pulses, the box color represents the wavelength of the pulse (red, 1.7$1.946 \mathrm{eV}$; green, typically $2.32 \mathrm{eV}$ ). The evolution of the charge and spin states of each defect and the transport electron are represented by solid arrows and are as annotated. The respective $1-\mu \mathrm{s}$ and 180 -ns time frames for ${ }^{14} \mathrm{~N}_{S}$ nuclear spin and transport electron spin relaxation are indicated by dashed arrows.

$\mathrm{NV}^{-}{ }_{-}{ }^{14} \mathrm{~N}_{S}$ pair: (1) initialization of the $\mathrm{NV}^{-}$spin and the charge and nuclear spin state of the ${ }^{14} \mathrm{~N}_{S}$, (2) preparation of the ${ }^{14} \mathrm{~N}_{S}$ electron spin into $|\psi\rangle$, and (3) photoionization of ${ }^{14} \mathrm{~N}_{S}$. In (1), the $\mathrm{NV}^{-}$electron spin is first spin polarized by a green optical pulse and the ${ }^{14} \mathrm{~N}_{S}$ is prepared into the neutral charge state by capturing an electron photoionized from a nearby ensemble of $\mathrm{N}_{S}$ centers by a red optical pulse. The $\mathrm{NV}^{-}$electron spin is then used to prepare the ${ }^{14} \mathrm{~N}_{S}$ nuclear spin into $m_{I}=0$ via microwave pulses $[3,4,8,9]$ before being repolarized by another green optical pulse. During this second green pulse, the ${ }^{14} \mathrm{~N}_{S}$ may photoionize, so a second red pulse and diffusion-capture process must occur to reprepare ${ }^{14} \mathrm{~N}_{S}$ in the neutral state. Alternatively, if the $\mathrm{NV}^{-}$nuclear spin is also initialized during the first initialization pulse using a projective technique $[10,47,48]$, then the nuclear spin and microwave pulses can be later used to reinitialize the $\mathrm{NV}^{-}$electron spin rather than a second optical pulse. The advantage of this alternative is that there is no interference with the ${ }^{14} \mathrm{~N}_{S}$ and, so, no requirement for charge reinitialization. In (2), the $\mathrm{NV}^{-}$electron spin and microwave pulses are used to prepare the ${ }^{14} \mathrm{~N}_{S}$ electron spin in $|\psi\rangle$. Finally, in (3), a red optical pulse is applied to photoionize the ${ }^{14} \mathrm{~N}_{S}$ and eject the prepared electron spin into the conduction band. Given the hyperfine dephasing of the ${ }^{14} \mathrm{~N}_{S}$ electron spin, steps (2) and (3) must be completed within $\sim 1 \mu$ s of the preparation of the ${ }^{14} \mathrm{~N}_{S}$ nuclear spin in (1). This can be comfortably achieved if the $\mathrm{NV}^{-}{ }_{-}^{14} \mathrm{~N}_{S}$ dipolar coupling is $\sim 10 \mathrm{MHz}$, so that the $\mathrm{NV}^{-}{ }_{-}^{14} \mathrm{~N}_{S}$ twoqubit gates that are used in (2) to prepare the ${ }^{14} \mathrm{~N}_{S}$ electron spin are limited to $\sim 100 \mathrm{~ns}$.

There are three steps to capture and optical detection [see Fig. 2(b)]: (1) initialization of the $\mathrm{NV}^{-}$spin and the charge and nuclear spin state of the ${ }^{14} \mathrm{~N}_{S}$, (2) capture of the electron by the ${ }^{14} \mathrm{~N}_{S}$, and (3) optical readout of the ${ }^{14} \mathrm{~N}_{S}$ electron spin via the $\mathrm{NV}^{-}$spin. Step (1) is as per photoionization, except the ${ }^{14} \mathrm{~N}_{S}$ is not recharged at the end and left in the positive charge state. In step (2), there is first a wait time for the electron to be photoionized, transported, and captured. In step (3), the ${ }^{14} \mathrm{~N}_{S}$ electron spin is readout by the $\mathrm{NV}^{-}$spin via two-qubit gates realized by microwave pulses and a final green optical pulse. As per photoionized, steps (2) and (3) must be completed within $\sim 1 \mu$ s of the time that the ${ }^{14} \mathrm{~N}_{S}$ nuclear spin is prepared in step (1). However, this is not the most restrictive time. The most restrictive time is $\sim 180 \mathrm{~ns}$ for transport and capture that is set by the intrinsic spin dephasing time of diamond. As discussed previously, the capture rate is proportional to the electron probability density at the ${ }^{14} \mathrm{~N}_{S}$. To achieve a capture time of $\sim 100 \mathrm{~ns}$, this probability density must be $\sim 50 \mu \mathrm{m}^{-3}$, which roughly corresponds to confining the electron to a cube with side lengths $\sim 270 \mathrm{~nm}$. Therefore, the transport of the electron must occur over $<80 \mathrm{~ns}$ and spatially controlled to this degree to achieve the capture within the total $180 \mathrm{~ns}$. This significant challenge is addressed in the next section.

Another important question that we have yet to address is whether it is possible for a free electron, other than the one prepared and photoionized, to be captured in error. In highpurity diamond, such free electrons can come only from background $\mathrm{N}_{S}$ impurities. If the $\mathrm{N}_{S}$ impurity of the diamond is comparable to or better than the attainable $\sim 10 \mu \mathrm{m}^{-3}$ [35], then given the $\sim 1.7-\mathrm{eV}$ depth of the $\mathrm{N}_{S}$ donor level, Fermi-Dirac statistics imply that there are essentially $\sim 0$ free electrons thermally excited to the conduction band. It also means that there will be $<0.25$ free electrons photoionized during the optical pulses (assuming a diffraction-limited spot of $\sim 300 \mathrm{~nm}$ in diameter). Hence, we surmise that the chance of capturing a spurious electron, instead of the one that is prepared and injected, can be made negligibly small. 


\section{SPIN TRANSPORT}

When the electron occupies a Bloch spin orbital near the conduction band minimum, its spin is decoupled from its orbital degrees of freedom and evolves according to the pure Zeeman spin Hamiltonian $H_{\text {cond }}=\gamma_{e} s_{z} B$. As described previously, this decoupling is due to the absence of spin-orbit interaction in the $\Delta_{1}$ conduction band. Furthermore, the electron $g$ factor in the $\Delta_{1}$ band is close to the free-electron value (within $\sim 10^{-3}$ ) because the interband spin-orbit interaction is small $(\sim 6 \mathrm{meV})$ compared with the interband energy separation $(\sim 5 \mathrm{eV})$ [56]. Since $H_{\text {cond }}$ is identical to Eq. (3) (when the ${ }^{14} \mathrm{~N}_{S}$ nuclear spin is correctly prepared), the electron does not accumulate a relative phase during its time in the Bloch spin orbital. Consequently, in the absence of impurities and magnetic field inhomogeneities, spin dephasing during transport is limited to the intrinsic Elliot-Yafet spin-relaxation mechanism of diamond.

The Elliot-Yafet mechanism is a mixture of interband spin-orbit interaction and momentum scattering by phonons [33,39]. The mechanism is dominant in solids with inversion symmetry and is particularly weak in diamond due to its small spin-orbit interaction, weak momentum scattering, and large band separations. Ab initio calculations predict that the Elliot-Yafet relaxation time in diamond at room temperature is the longest of any solid, $T_{2} \sim T_{1} \sim 180 \mathrm{~ns}$ [33]. As discussed in the previous section, this defines the maximum time allowed for transport and capture and we allocate $\sim 80$ and $\sim 100 \mathrm{ns,}$ respectively.

Given the decoupling of the electron's orbital and spin degrees of freedom, only its orbital degrees of freedom need to be considered in a model of its transport. For weak applied electric fields $\vec{E}_{\text {ap }}$ where transport occurs over time scales much longer than momentum and energy relaxation within the conduction band ( $\sim 1 \mathrm{ps})$, a drift-diffusion model of the injected electron's probability density $\rho(\vec{r}, t)$ may be adopted [35,61]:

$$
\begin{aligned}
\frac{\partial \rho}{\partial t}= & \vec{\nabla} \cdot\left(D_{n} \vec{\nabla} \rho+\mu_{n} \rho \vec{E}\right) \\
& +k_{I}(t) N_{I} \delta\left(\vec{r}-\vec{r}_{I}\right)-\rho k_{C}\left[\left(1-N_{C}\right) \delta\left(\vec{r}-\vec{r}_{C}\right)\right. \\
& \left.+\left(1-N_{I}\right) \delta\left(\vec{r}-\vec{r}_{I}\right)\right] \\
\frac{d N_{I}}{d t}= & -k_{I}(t) N_{I}+\rho k_{C}\left(1-N_{I}\right), \\
\frac{d N_{C}}{d t}= & \rho k_{C}\left(1-N_{C}\right),
\end{aligned}
$$

where $\mu_{n}=450 \mu \mathrm{m}^{2} / \mathrm{Vns}$ is the room-temperature electron mobility in high-purity diamond [62], $D_{n}=$ $\mu_{n} k_{B} T / e=11 \mu \mathrm{m}^{2} / \mathrm{ns}$ is the room-temperature electron diffusion constant, $e$ is the fundamental charge, $k_{I}(t)$ is the photoionization rate of the ionizing ${ }^{14} \mathrm{~N}_{S}$ center located at
$\vec{r}_{I}$ during the optical injection pulse, $k_{C}=\sigma_{\text {cap }} \sqrt{k_{B} T / m}$ is the (re)capture rate per unit electron density of the (ionizing) capturing ${ }^{14} \mathrm{~N}_{S}$ center located at $\left(\vec{r}_{I}\right) \vec{r}_{C}, N_{I}$ and $N_{C}$ are the probabilities that the electron is occupying the ionizing and capturing centers, respectively,

$$
\vec{E}=\vec{E}_{\mathrm{ap}}-\frac{e}{4 \pi \epsilon} \vec{\nabla}\left[\frac{1-N_{I}}{\left|\vec{r}-\vec{r}_{I}\right|}+\frac{1-N_{C}}{\left|\vec{r}-\vec{r}_{C}\right|}\right]
$$

is the total electric field including contributions from the charged centers, and $\epsilon$ is the electric permittivity of diamond.

Consider the simple transport architecture depicted in Fig. 3 that consists of two surface electrodes on bulk diamond. For simplicity, we ignore the finite photoionization time and slow capture processes and make the approximation $\vec{E} \approx \vec{E}_{\text {ap }} \approx-E \hat{z}$ [63]. In this case, the drift-diffusion model reduces to $\partial \rho / \partial t=D_{n} \nabla^{2} \rho-\mu_{n} E \partial \rho / \partial z$. The initial condition can be defined by approximating the electron probability density by a Gaussian $\rho(\vec{r}, 0)=$ $e^{-\left|\vec{r}-\vec{r}_{I}\right|^{2} / 2 w^{2}} / \sqrt{2 \pi w^{2}}$ centered at $\vec{r}_{I}=x_{I} \hat{x}$ and with width $w$. This approximate model has the separable solution $\rho=X(x, t) Y(y, t) Z(z, t)$, where

$$
\begin{aligned}
& X(x, t)=\frac{1}{\sqrt{4 \pi D_{n} t^{*}}}\left[e^{-\left(x+x_{I}\right)^{2} / 4 D_{n} t^{*}}+e^{-\left(x-x_{I}\right)^{2} / 4 D_{n} t^{*}}\right], \\
& Y(y, t)=\frac{1}{\sqrt{4 \pi D_{n} t^{*}}} e^{-y^{2} / 4 D_{n} t^{*}}, \\
& Z(z, t)=\frac{1}{\sqrt{4 \pi D_{n} t^{*}}} e^{-z^{2} / 4 D_{n} t^{*}} e^{\left(\mu_{n} E / 4 D\right)\left(2 z-\mu_{n} E t\right)},
\end{aligned}
$$

$t^{*}=t+w^{2} / 2 D_{n}$, and the zero flux $\left(D_{n} \vec{\nabla} \rho+\mu_{n} \rho \vec{E}\right) \cdot \hat{n}=$ 0 boundary condition is enforced at the surface with unit normal vector $\hat{n}$. For $\sqrt{2 D_{n} t} \ll x_{I}$, the mean position of $\rho$ is $\langle\vec{r}\rangle \approx x_{I} \hat{x}+\mu_{n} E t \hat{z}$ and its width $\left[\left\langle(x-\langle x\rangle)^{2}\right\rangle\right]^{1 / 2}=$ $\left[\left\langle(y-\langle y\rangle)^{2}\right\rangle\right]^{1 / 2}=\left[\left\langle(z-\langle z\rangle)^{2}\right\rangle\right]^{1 / 2} \approx \sqrt{2 D_{n} t^{*}}$ is isotropic and, for $D_{n} t \gg w^{2}$, approximately equal to $\sqrt{2 D_{n} t}$. So, on average, the electron is transported a distance $\mu_{n} E t$ and freely diffused over a volume with radius $\sqrt{2 D_{n} t}$. Drawing upon the conclusions of the previous section, to achieve capture within the allocated $\sim 100 \mathrm{~ns}, \rho$ must be centered at $\vec{r}_{C}$ (i.e., $\mu_{n} E t=d$, where $\vec{r}_{C}=\vec{r}_{I}+d \hat{z}$ ) and have a radius $\sqrt{2 D_{n} t}<135 \mathrm{~nm}$ for a period of time $\sim 100 \mathrm{~ns}$. However, after just $\sim 1 \mathrm{ps}$, the radius will be $>135 \mathrm{~nm}$ [64], and so we can immediately conclude that this simple transport architecture cannot meet the requirements for capture because it does not adequately confine the diffusion of the electron probability density.

We can conceive of two strategies to confine the electron diffusion: (1) apply confining electric fields via additional surface electrodes or (2) employ a diamond nanowire. There are two approaches to strategy (1): (A) use transverse electrodes to tightly focus the electron diffusion in the 


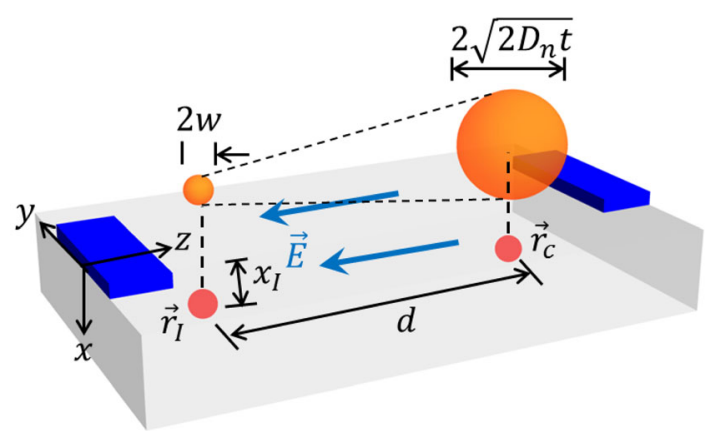

(a)

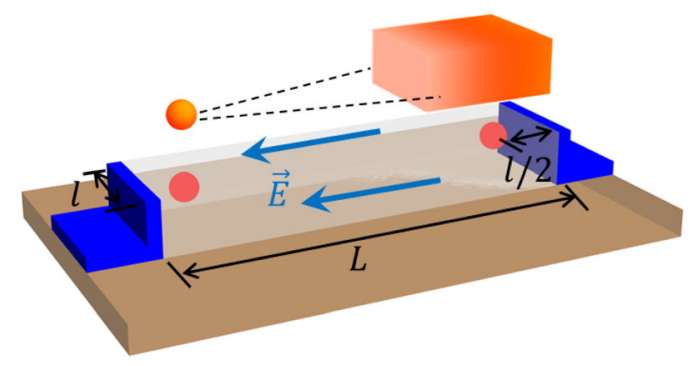

(b)

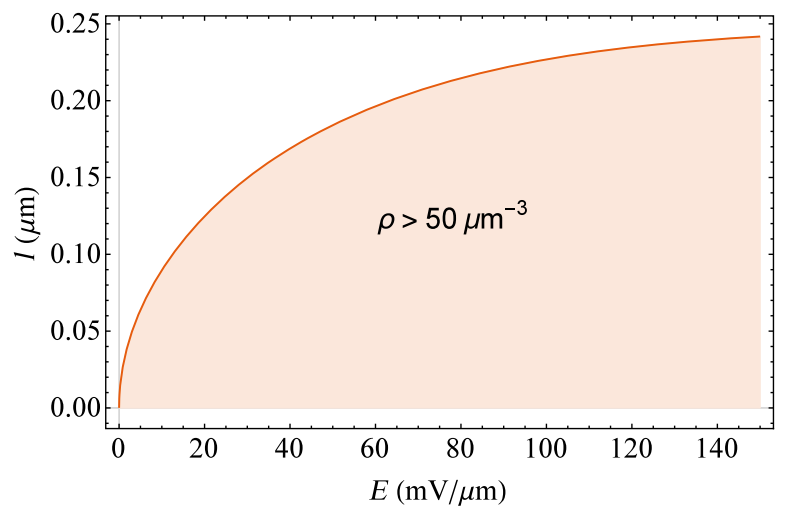

(c)

FIG. 3. (a) A simple transport architecture consisting of two surface electrodes (dark blue) on bulk diamond (gray). (b) Example architecture for transport via a nanowire (gray) on top of a substrate (brown) with end electrodes (dark blue). In (a) and (b), the ionizing and capture centers are depicted as red spheres and the initial and final electron probability densities are depicted above in orange. All other labels are as described in the text. (c) The shaded region defines the nanowire dimension $l$ and applied electric field $E$ where the electron probability density at the capture center is the required $\rho>50 \mu \mathrm{m}^{-3}$ to yield a capture time $<100 \mathrm{~ns}$.

transverse directions to achieve very high electron density at the capture center or (B) trap the electron using an electrostatic trap (e.g., a quadrupole trap [65]) and move the trap site from the ionizing to capture centers over the allocated $\sim 80 \mathrm{~ns}$. A significant undertaking of device design is clearly required to properly assess strategy (1). Here, we leave this undertaking for future work and instead focus on strategy (2).
Consider the rectangular diamond nanowire with dimension $l \times l \times L$ sketched in Fig. 3. The side and end surfaces of the wire confine the electron probability density, such that after sufficient time, $t \gg l^{2} / D_{n}$ and $L / \mu_{n} E$, it reaches the static distribution

$$
\rho(\vec{r}) \approx \frac{\mu_{n} E}{D_{n} l^{2}} e^{-\left(\mu_{n} E / D_{n}\right)(L-z)},
$$

where it is assumed that $\mu_{n} E L / D_{n} \gg 1 . \rho$ is thus uniformly distributed over the transverse wire cross section and confined to the wire end face by an exponential distribution with decay constant $\mu_{n} E / D_{n}$. Defining the distance from the capture center to the end face to be $\left(L-z_{C}\right)=l / 2$, Fig. 3(c) plots the region in the parameter space $\{E, l\}$ where the electron probability density $\rho>50 \mu \mathrm{m}^{-3}$ is sufficiently large to yield a capture time $<100 \mathrm{~ns}$. For $l=0.2 \mu \mathrm{m}$, the applied electric field must be $E>63 \mathrm{mV} / \mu \mathrm{m}$, which is achievable [66]. In such electric fields, the center of the electron density is transported at a speed $\mu_{n} E>28 \mu \mathrm{m} / \mathrm{ns}$ and can, in principle, be transported $>2 \mathrm{~mm}$ during the allocated $\sim 80 \mathrm{~ns}$. This is clearly more than adequate for a scalable device. Given that the technology already exists to fabricate diamond nanowires of these dimensions that contain $\mathrm{NV}$ and $\mathrm{N}_{S}$ centers [67-70], diamond nanowires appear to be a very promising strategy to control spin transport.

This encouraging conclusion is, however, conditional on two assumptions that require further investigation: (1) the zero-flux boundary condition is strictly enforced at the diamond surface so that there is perfect confinement of the electron to the nanowire's interior and (2) spin relaxation during transport has not been altered by the structure and surfaces of the nanowire. Partial failure of these assumptions will result in electron losses and increased spin relaxation, respectively, and reduce the maximum attainable transport distance below the ideal estimate of $2 \mathrm{~mm}$.

Assumption (1) pertains to the termination and quality of the nanowire surfaces. Surface defects and impurities can act as electron traps and so must be avoided. Nanowire surfaces exposed to air or vacuum must have a positive electron affinity in order to avoid loss of the electron. Oxygen- and nitrogen-terminated diamond surfaces are known to have positive electron affinities [71]. The latter, in particular, is predicted to have low densities of paramagnetic defects and therefore is more suited to spinrelated applications [71]. Alternatively, it is possible that surface emission can be avoided by embedding the nanowire in another semiconductor or a dielectric, whose electron affinity is much smaller than diamond's. To assess the validity of assumption (1), future work should explore the yet-to-be-studied electronic properties of diamond nanowires and their associated surface effects.

Assumption (2) pertains to the increase of spin relaxation by paramagnetic surface defects or the emergence of 
another transport relaxation mechanism other than the Elliot-Yafet mechanism. While the former can, in principle, be controlled by fabrication precision and choice of surface termination, the latter is a more fundamental question. If the geometry of the nanowire sufficiently perturbs the inversion symmetry properties of the conduction band Bloch orbitals, then the D'yakonov-Perel' spin-relaxation mechanism may emerge [39]. However, as observed in Ref. [72], as long as the nanowire's width is smaller than or comparable to the electron mean-free path, the D'yakonovPerel' mechanism is suppressed. Since the electron meanfree path in diamond at room temperature $\mu_{n} \sqrt{m k_{B} T} / e \sim$ $0.17 \mu \mathrm{m}$ is comparable with the relevant nanowire widths, we expect that the increased spin relaxation due to the D'yakonov-Perel' mechanism will be negligible. Having said this, future $a b$ initio studies are really needed to properly assess the emergence of the D'yakonov-Perel' mechanism in diamond nanowires.

\section{ROOM-TEMPERATURE SPIN QUANTUM BUS}

Based on the conclusions of the previous sections, we propose that a room-temperature on-chip spin quantum bus can be constructed in diamond by connecting two defect spin clusters by a diamond nanowire [see Fig. 4(a)]. Each spin cluster contains at least a NV center, a ${ }^{14} \mathrm{~N}_{S}$ donor center, and a logic spin qubit (either the $\mathrm{N}$ nuclear spin of the NV center, a ${ }^{13} \mathrm{C}$ isotopic impurity, or another $\mathrm{NV}$ center). The NV center acts as a fast local bus to prepare, control, and readout the logic and ${ }^{14} \mathrm{~N}_{S}$ spins with high fidelity. The logic spin is chosen to have a long coherence time to minimize errors. The ${ }^{14} \mathrm{~N}_{S}$ center is included to facilitate spin-coherent electron transmission between clusters via photoionization, transport, and capture, as outlined in previous sections. In order to recharge the ${ }^{14} \mathrm{~N}_{S}$ center on demand, each spin cluster is accompanied by a nearby ensemble of $\mathrm{N}_{S}$ centers to act as donors upon photoionization. Surface electrodes are used to control the spin transport between the clusters and the recharge process. The NV centers of the clusters have the same orientation, which can be achieved via preferential growth techniques [73], and the applied magnetic field is aligned with this orientation.

In a scalable design, similar to the one outlined in Ref. [38], the spin clusters are located at the nodes of a network of diamond nanowires. Pursuant to the discussion of the previous section, the surface electrodes must also be used to apply an electrostatic trap [e.g., via a quadrupole configuration as per Fig. 4(a)] at the node in order to confine the transport electron and achieve the required capture rate. It is possible that there are other network designs where the spin clusters are not located at nodes, but somewhere where the nanostructured diamond can offer confinement of the transport electron without the need for electrostatic traps. While the maximum distance between

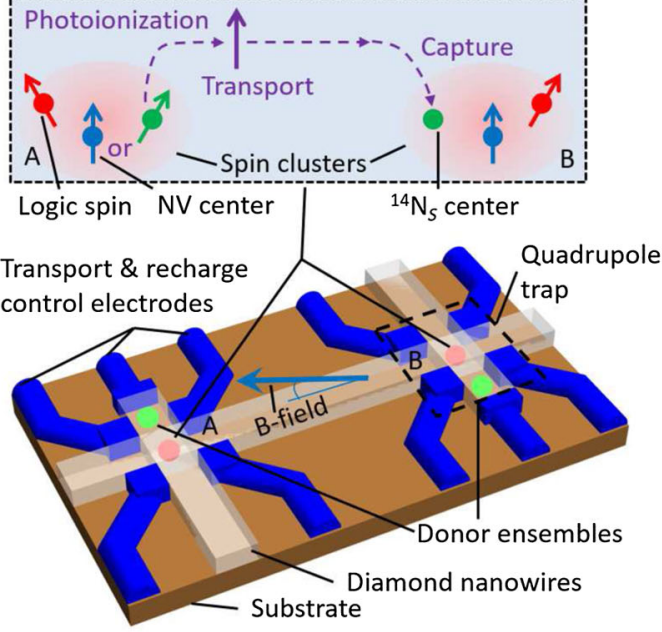

(a)

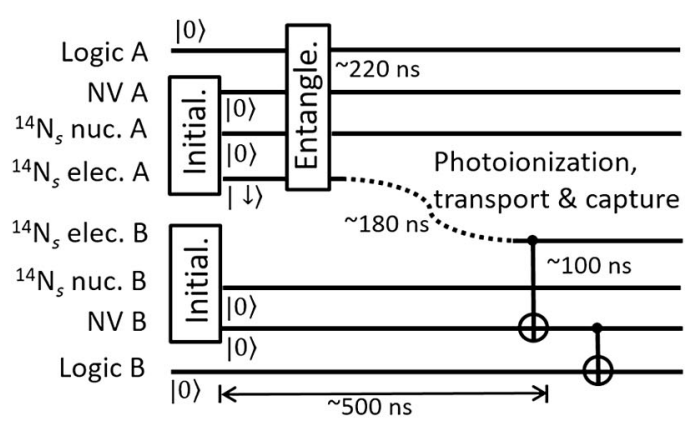

(b)

FIG. 4. (a) Sketch of two nodes of the proposed spin quantum bus network constructed from diamond nanowires (white) on top of a substrate (brown). Spin clusters A and B (red) are located at the nodes and contain logic (either the $\mathrm{N}$ nuclear spin of the $\mathrm{NV}$ center or a ${ }^{13} \mathrm{C}$ isotopic impurity), $\mathrm{NV}$, and ${ }^{14} \mathrm{~N}_{S}$ spins. Nearby ensembles of $\mathrm{N}_{S}$ centers (green) for recharging the ${ }^{14} \mathrm{~N}_{S}$ centers in the clusters are located in compartments next to the nodes. Surface electrodes (blue) are used to control electron transport between clusters and the recharge process. At each node, four of the electrodes are arranged in a quadruple configuration to aid in trapping the transport electron at the node. A magnetic field ( $B$ field) defines the spin quantization axis and is aligned with the (assumed identical) axes of the NV centers. For clarity, optical, microwave, and magnetic control structures have not been included in the sketch. (b) The proposed protocol to generate entanglement between the logic qubits located in clusters A and B. See text for details of the protocol.

spin clusters is important to assess the range of connections between spin clusters, the minimum distance is important to assess the ultimate scalability of the device (i.e., how many clusters can be integrated on a single chip). The maximum distance is as determined in the previous section to be $\sim 2 \mathrm{~mm}$. The minimum distance is determined by the dimensions of the electrodes and diamond nanowires $(\sim 0.2 \mu \mathrm{m})$ as well as the ability to selectively apply microwave control pulses to the clusters. If the clusters 
are too close to be addressed by individual microwave microstructures (i.e., $<1 \mu \mathrm{m}$ ), then the latter requires the application of a magnetic field gradient during pulses (removed during transport to avoid spin dephasing) by a magnetic microstructure. Sufficient gradients $(>10 \mathrm{~T} / \mu \mathrm{m})$ to selectively address clusters $\sim 0.2 \mu \mathrm{m}$ apart can be achieved using magnetic resonance force microscopy tips [74]. However, we leave the precise implementation of such tips as a question for future work and instead conservatively estimate a minimum cluster separation of $>1 \mu \mathrm{m}$.

In previous sections, we discuss how this design of a spin quantum bus can be used to coherently transmit a spin state between two spin clusters. The other important function of a quantum bus is to generate entanglement between distant logic qubits. In our design, entanglement is generated between two distant logic qubits, located in spin clusters $\mathrm{A}$ and $\mathrm{B}$, via the coherent transport of an entangled spin and entanglement swapping operations. In Fig. 4, we outline a protocol to generate a maximally entangled Bell state of logic qubits $\mathrm{A}$ and $\mathrm{B}$. The protocol begins with the initialization of the $\mathrm{NV}$ and ${ }^{14} \mathrm{~N}_{S}$ centers in the two clusters as defined in Sec. III. The desired Bell state is generated between logic $A$ and ${ }^{14} \mathrm{~N}_{S}$ electron spin $\mathrm{A}$ via a fast entanglement gate mediated by NV A, such as CPHASE $[10,75]$. Photoionization, transport, and capture proceeds as defined in Secs. III and IV and transfers the transport electron from ${ }^{14} \mathrm{~N}_{S}$ A to ${ }^{14} \mathrm{~N}_{S}$ B. Finally, the entanglement is swapped from the transport electron at ${ }^{14} \mathrm{~N}_{S} \mathrm{~B}$ to $\operatorname{logic} \mathrm{B}$ via a series of CNOT gates and NV B.

The only complication that this entanglement protocol adds to the ones already discussed in Secs. III and IV is that the entanglement gate must also be performed within the $\sim 1-\mu$ s period extending from initialization of the ${ }^{14} \mathrm{~N}_{S}$ nuclear spins to the final operation on the transport electron [the CNOT gate with NV B in Fig. 4(b)]. Since the complete initialization sequence defined in Sec. III includes 500 ns after the initialization of the ${ }^{14} \mathrm{~N}_{S}$ nuclear spins, there is $\sim 500 \mathrm{~ns}$ left for the gate operations and spin transport in Fig. 4(b). Given the $\mathrm{NV}_{-}{ }^{14} \mathrm{~N}_{S}$ gate operation times and spin transport times already discussed, the entanglement gate must be completed within $\sim 220$ ns. Assuming a NV ${ }^{14} \mathrm{~N}_{S}$ coupling of $\sim 10 \mathrm{MHz}$, the entanglement gate can be completed within $\sim 220 \mathrm{~ns}$ as long as the NV-logic qubit coupling is also $>10 \mathrm{MHz}$. This is attainable for ${ }^{13} \mathrm{C}$ logic qubits [6]. Hence, we conclude that, in light of all of our considerations, it is feasible to realize the entanglement generation protocol of Fig. 4(b).

It remains to estimate the fidelity of the entanglement generated by the protocol, which is given by the product of the fidelities of each of the spin cluster initialization, measurement, one- and two-qubit gates, and transport operations. Initialization (>99\%), measurement (>99\%), and one- (>99\%) and two-qubit (>90\%) gate operation fidelities at or approaching fault-tolerant thresholds (> 99\%) have been previously demonstrated by diamond spin clusters [10]. Pursuant to the discussion in Sec. IV, the transport fidelity is primarily determined by the transport spin relaxation $\sim e^{-T_{\mathrm{tr}} / T_{2}}$, where $T_{\mathrm{tr}}$ is the transport and capture time and $T_{2} \sim 180 \mathrm{~ns}$ is the transport spin lifetime. As the protocol has been outlined above, the transport time is taken to be $T_{\text {tr }} \sim T_{2}$, and so the transport fidelity is $\sim 37 \%$. Thus, the entanglement fidelity is $\approx 37 \%$, which is far from thresholds for fault-tolerant quantum computing. Only if the transport time can be reduced to $<2$ ns through nanoelectrode and nanowire engineering can the protocol approach the required thresholds at room temperature. Alternatively, there may be a heralded entanglement protocol at room temperature that we are yet to identify that more simply meets the thresholds. The protocol and architecture as outlined may, however, meet the thresholds at cryogenic temperatures, where it is projected that $T_{2}>10 \mu \mathrm{s}$ [33]. In which case, it will be competitive with existing cryogenic optical quantum bus realizations, particularly since it potentially has an entanglement generation rate of $\sim 100 \mathrm{kHz}$ compared to the demonstrated $\sim 10^{-3} \mathrm{~Hz}$ for the optical approach [21]. However, it should be highlighted that the optically generated entanglement is heralded.

\section{CONCLUSION}

In this paper, we explore an alternate approach to realizing a room-temperature spin quantum bus in diamond, a major unsolved problem in the development of scalable diamond QIP devices. Our approach is motivated by the unique properties of diamond paramagnetic defects and the predictions of extreme spin transport in diamond. By reviewing the properties of the $\mathrm{NV}, \mathrm{N}_{S}$, and $\mathrm{P}_{S}$ centers, we identify novel photoionization and capture mechanisms. After assessing the mechanisms, we conclude that $\mathrm{NV}-{ }^{14} \mathrm{~N}_{S}$ pairs are the best option for spin-coherent photoionization and capture. We explore spin transport in diamond by applying a drift-diffusion model of electron transport to simple architectures. It becomes clear that adequate confinement of the transport electron, so that the required capture rates can be achieved, is the principal problem that must be overcome in order to realize spin transport between discrete spin clusters. We identify that diamond nanowires are a promising solution and that they may be combined with other strategies, such as electrostatic traps, in scalable designs of networked quantum buses. We, finally, propose how such nanowire quantum buses can coherently transmit spin states between and generate entanglement of distant spin clusters.

The main purpose of this paper is to stimulate investigation of this alternate approach. As we highlight throughout our discussion, there are many aspects that require further experimental and theoretical study. These aspects range from the fundamental properties of the defect centers to the electrical properties of diamond nanowires. As this future work progresses, we expect that the 
preliminary ideas that we outline here will substantially evolve.

\section{ACKNOWLEDGMENTS}

This work was supported by the Australian Research Council under the Discovery Project scheme (DP120102232), the National Science Foundation (NSF1314205), and a grant (No. M-ERA.NET-1/2015) from the Research Council of Lithuania. We wish to thank A. Stacey, J. Meijer, K. Ganesan, A. Greentree, L. C. L. Hollenberg, and $\mathrm{S}$. Prawer for many useful discussions.

\section{APPENDIX A: PHOTOIONIZATION PROCESS OF THE NV- CENTER}

$\mathrm{NV}^{-}$can be photoionized into $\mathrm{NV}^{0}$ by ejecting an electron into the diamond conduction band via the absorption of one photon with energy $>2.6 \mathrm{eV}$ [42] or by the successive absorption of two photons with energies $>$ $1.946 \mathrm{eV}$ (see Fig. 5) [78]. In the latter mechanism, the first photon excites $\mathrm{NV}^{-}$to its optical excited state and the second photon ejects the electron into the conduction band. Detection of the photocurrent produced by $\mathrm{NV}^{-}$photoionization was reported recently [79]. $\mathrm{NV}^{0}$ can be photoconverted back to $\mathrm{NV}^{-}$by a similar mechanism involving the ejection of a hole into the diamond valence band and the absorption of one photon with energy $>2.94 \mathrm{eV}$ or the successive absorption of two photons with energies $>$ $2.156 \mathrm{eV}[42,78]$. These back photoconversion mechanisms allow the desired negative charge state to be prepared on demand.

In the simplest picture, if a $\mathrm{NV}^{-}$center is optically prepared into the $m_{s}=+1$ spin state (or equivalently the $m_{s}=-1$ state) and then photoionized by the absorption of one photon with energy near threshold, $\sim 2.6 \mathrm{eV}$, then a spin-up (spin-down) electron is ejected into the conduction band with $100 \%$ probability. If the $\mathrm{NV}^{-}$center is instead photoionized by the successive absorption of two photons with energy $>1.946 \mathrm{eV}$, then a spin-up (spin-down) electron is ejected into the conduction band with a much reduced $66 \%$ probability because there is equal chance that the electron was ejected from any one of the three occupied (two spin-up and one spin-down) $e$ spin orbitals in the ${ }^{3} E$ optical excited state [81]. However, this latter picture ignores the possibility that the $\mathrm{NV}^{-}$center may, instead of absorbing a second photon and ionizing, decay back to the ground state. Owing to the optical spin-polarization mechanism, this decay may flip the $\mathrm{NV}^{-}$spin to the $m_{s}=0$ state, which reduces the probability that a spin-up (spindown) electron is ejected during a subsequent photoionization event. It also ignores the possibility that absorption of the second photon from the optical excited state may ionize the electron $0.5 \mathrm{eV}$ above threshold, where spin mixing may occur in the final conduction band orbitals [80], which will also reduce the probability. The low $66 \%$

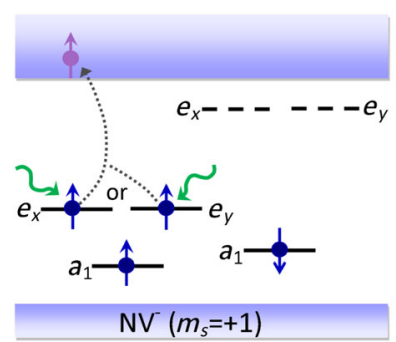

(a)

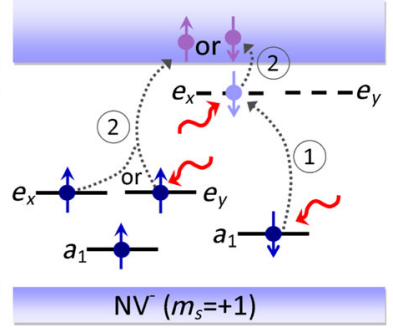

(b)

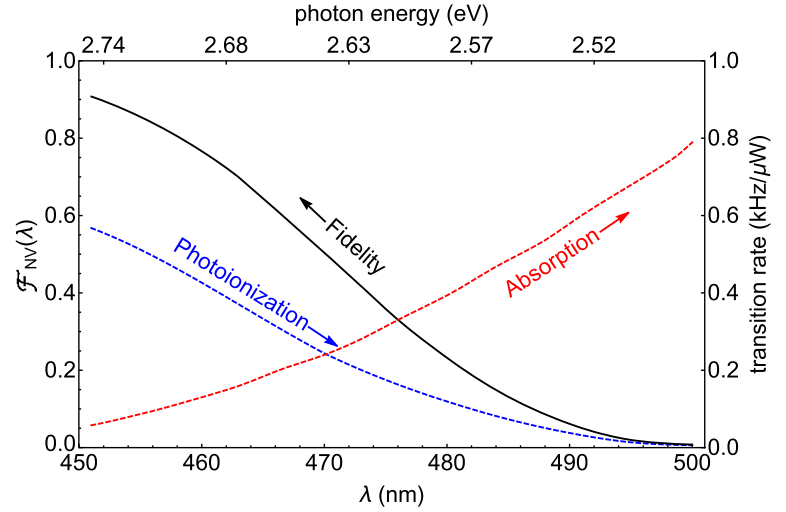

(c)
FIG. 5. The one- (a) and two-photon (b) ionization mechanisms of $\mathrm{NV}^{-}$. The depicted mechanisms correspond to photionization from the $m_{s}=+1$ spin state of the $\mathrm{NV}^{-}$ground ${ }^{3} A_{2}$ level. In (a), one photon with energy $>2.6 \mathrm{eV}$ ejects an electron from the spin-up $e$ spin orbitals of $\mathrm{NV}^{-}$into the conduction band. In (b), one photon with energy $>1.946 \mathrm{eV}$ promotes an electron from the occupied spin-down $a_{1}$ spin orbital to one of the unoccupied spin-down $e$ spin orbitals (i.e., a transition from ${ }^{3} A_{2}$ to ${ }^{3} E$ ). A second photon with energy $>1.946 \mathrm{eV}$ ejects an electron from either the spin-up $e$ spin orbitals or the occupied spin-down $e$ spin orbital into the conduction band. In (a) and (b), occupied or unoccupied spin orbitals are depicted as solid or dashed lines, filled circles represent electrons and solid arrows denote spin-up or spin-down electrons, wavelike arrows denote photons. Panel (c) depicts the estimated ultimate photoionization fidelity $\mathcal{F}_{\mathrm{NV}}(\lambda)$ for one-photon photoionization (solid black line, left axis) [76] as well as the one-photon photoionization [42] (dashed blue line, right axis) and optical absorption [77] (dashed red line, right axis) transition rates per unit optical power (in the confocal arrangement described in Ref. [42]).

probability plus these further reductions essentially eliminate the two-photon photoionization mechanism as an option.

Noting that the differing spin-spin interactions in the $\mathrm{NV}^{-}$ground and optically excited states means that the electron spin dephases rapidly if the center is optically excited, the ultimate fidelity $\mathcal{F}_{\mathrm{NV}}$ for spin-coherent onephoton photoionization is determined by the relative probability that, upon illumination, the center absorbs a photon and photoionizes or absorbs a photon and transitions to the optical excited state. The fidelity takes the simple expression 


$$
\mathcal{F}_{\mathrm{NV}}(\lambda)=\frac{\sigma_{\text {ion }}(\lambda)}{\sigma_{\text {ion }}(\lambda)+\sigma_{\text {opt }}(\lambda)},
$$

where $\lambda$ is the optical wavelength, and $\sigma_{\text {ion }}$ and $\sigma_{\text {opt }}$ are the wavelength-dependent photoionization and optical absorption cross sections, respectively. In Fig. 5, we plot an estimate [76] of $\mathcal{F}_{\mathrm{NV}}(\lambda)$ based on data from Ref. [42] for the one-photon photoionization rate $\propto \sigma_{\text {ion }}(\lambda)$ and the wellknown $\mathrm{NV}^{-}$optical absorption spectrum $\propto \sigma_{\text {opt }}(\lambda)$ [77]. As can be seen, $\sigma_{\text {opt }}(\lambda)$ decays towards shorter wavelength, whereas $\sigma_{\text {ion }}(\lambda)$ grows. $\mathcal{F}_{\mathrm{NV}}(\lambda)$ therefore increases towards shorter wavelength until it is ultimately limited to $\sim 90 \%$ by the onset at $\lambda \sim 452 \mathrm{~nm}$ (photon energy $\sim 2.74 \mathrm{eV}$ ) [82] of two-photon indirect excitation of electrons to the conduction band from the diamond valence band. This interband excitation will generate many conduction electrons with unpolarized spins, which will swamp the spin-polarized electrons photoionized from $\mathrm{NV}^{-}$. While $\sim 90 \%$ fidelity may be useful for applications in spintronics where spin polarization and not spin coherence is required, it is too low for the operation of a spin quantum bus. Thus, we completely rule out $\mathrm{NV}^{-}$as a source of spin-coherent photoionized electrons.

\section{APPENDIX B: CONSIDERATION OF THE $P_{S}$ CENTER}

In this Appendix, we consider the other prevalent paramagnetic donor center in diamond, the substitutional phosphorus $\mathrm{P}_{S}$ center, in a similar manner to the $\mathrm{N}_{S}$ center. While this is a worthy exercise, our ultimate conclusion is that $\mathrm{P}_{S}$ is unsuitable for spin-coherent electron photoionization and capture.

The $\mathrm{P}_{S}$ center is a comparatively shallow donor in diamond with six defect levels within $0.6 \mathrm{eV}$ of the conduction band [83-85]. As per the $\mathrm{N}_{S}$ center, in the neutral charge state $\mathrm{P}_{S}^{0}$, one of these levels is occupied by an electron, whereas in the positive charge state $\mathrm{P}_{S}^{+}$, they are unoccupied [83-85]. It may be possible for NV-P ${ }_{S}$ clusters to be created in a similar way as NV-N ${ }_{S}$ clusters via N-P codoping or coimplantation. However, we note that there are well-known difficulties in the incorporation of phosphorus into diamond that reduce the yield of phosphorus atoms occupying substitutional, rather than split-vacancy, sites [86-88]. The latter is problematic because it is a deep acceptor rather than a donor.

Similar to $\mathrm{N}_{S}, \mathrm{P}_{S}^{0}$ can be photoionized into $\mathrm{P}_{S}^{+}$via absorption of one photon of energy $>0.6 \mathrm{eV} . \mathrm{P}_{S}^{+}$may be converted back to $\mathrm{P}_{S}^{0}$ by capture of either a conduction band electron via a nonradiative process (likely to be a cascade mechanism) or a valence band electron via absorption of a UV photon with energy $>4.8 \mathrm{eV}$. To our knowledge, the nonradiative electron capture cross section of $\mathrm{P}_{S}^{+}$has not been measured. Given that $\mathrm{N}_{S}$ is a deeper donor than $\mathrm{P}_{S}$, it is reasonable to expect that the cross section of $\mathrm{P}_{S}^{+}$is larger than that of $\mathrm{N}_{S}^{+}$and will, therefore, achieve faster capture rates for the same diffusing electron densities.

It is known from ESR studies that $\mathrm{P}_{S}^{0}$ is distorted to $D_{2 d}$ symmetry with $\langle 100\rangle$ orientation [83]. Unlike $\mathrm{N}_{S}^{0}$, this is known to be due to the Jahn-Teller effect. The ESR broadens with temperature and eventually becomes undetectable at $\sim 20 \mathrm{~K}[83,85]$. This broadening is most likely due to rapid reorientation via Jahn-Teller dynamics [85]. It is still an open question as to whether these dynamics reach the motionally narrowed limit at higher temperatures and, as a consequence, if $\mathrm{P}_{S}^{0}$ has a usable electronic spin at room temperature. This question should be answered by future ESR or NV DEER measurements.

If the motionally narrowed limit is achieved by room temperature, then the spin Hamiltonian of $\mathrm{P}_{S}^{0}$ will be similar to that of $\mathrm{N}_{S}^{0}$ [see Eq. (2)]. The spin parameters are $g_{e} \sim 2$, $g_{n}=2.26, I=1 / 2, A_{\|}=162 \mathrm{MHz}$, and $A_{\perp}=33.9 \mathrm{MHz}$ [83]. As per the arguments presented for ${ }^{15} \mathrm{~N}_{S}$, the large magnetic hyperfine interactions between the electron spin and the $I=1 / 2$ nuclear spin of $\mathrm{P}_{S}^{0}$ pose major problems for spin-coherent photoionization and capture. Hence, we similarly rule out $\mathrm{P}_{S}$ as a viable option for realizing a spin quantum bus, but note that it may be suitable for applications that simply require the production of spin-polarized conduction electrons.

[1] M. W. Doherty, N. B. Manson, P. Delaney, F. Jelezko, J. Wrachtrup, and L. C. L. Hollenberg, The Nitrogen-Vacancy Colour Centre in Diamond, Phys. Rep. 528, 1 (2013).

[2] G. Balasubramanian, P. Neumann, D. Twitchen, M. Markham, R. Kolesov, N. Mizuochi, J. Isoya, J. Achard, J. Beck, J. Tissler, V. Jacques, P. R. Hemmer, F. Jelezko, and J. Wrachtrup, Ultralong Spin Coherence Time in Isotopically Engineered Diamond, Nat. Mater. 8, 383 (2009).

[3] T. Gaebel, M. Domhan, I. Popa, C. Wittmann, P. Neumann, F. Jelezko, J. R. Rabeau, N. Stavrias, A. D. Greentree, S. Prawer, J. Meijer, J. Twamley, P. R. Hemmer, and J. Wrachtrup, Room-Temperature Coherent Coupling of Single Spins in Diamond, Nat. Phys. 2, 408 (2006).

[4] R. Hanson, F. M. Mendoza, R. J. Epstein, and D. D. Awschalom, Polarization and Readout of Coupled Single Spins in Diamond, Phys. Rev. Lett. 97, 087601 (2006).

[5] M. V. G. Dutt, L. Childress, L. Jiang, E. Togan, J. Maze, F. Jelezko, A.S. Zibrov, P. R. Hemmer, and M. D. Lukin, Quantum Register Based on Individual Electronic and Nuclear Spin Qubits in Diamond, Science 316, 1312 (2007).

[6] P. Neumann, N. Mizuochi, F. Rempp, P. Hemmer, H. Watanabe, S. Yamasaki, V. Jacques, T. Gaebel, F. Jelezko, and J. Wrachtrup, Multipartite Entanglement among Single Spins in Diamond, Science 320, 1326 (2008).

[7] P. Neumann, R. Kolesov, B. Naydenov, J. Beck, F. Rempp, M. Steiner, V. Jacques, G. Balasubramanian, M. L. Markham, D. J. Twitchen, S. Pezzagna, J. Meijer, J. Twamley, F. Jelezko, and J. Wrachtrup, Quantum Register 
Based on Coupled Electron Spins in a Room-Temperature Solid, Nat. Phys. 6, 249 (2010).

[8] F. Dolde, I. Jakobi, B. Naydenov, N. Zhao, S. Pezzagna, C. Trautmann, J. Meijer, P. Neumann, F. Jelezko, and J. Wrachtrup, Room-Temperature Entanglement between Single Defect Spins in Diamond, Nat. Phys. 9, 139 (2013).

[9] F. Dolde, V. Bergholm, Y. Wang, I. Jakobi, B. Naydenov, S. Pezzagna, J. Meijer, F. Jelezko, P. Neumann, T. SchulteHerbrüggen, J. Biamonte, and J. Wrachtrup, High-Fidelity Spin Entanglement Using Optimal Control, Nat. Commun. 5, 3371 (2014).

[10] G. Waldherr, Y. Wang, S. Zaiser, M. Jamali, T. SchulteHerbrüggen, H. Abe, T. Ohshima, J. Isoya, J. F. Du, P. Neumann, and J. Wrachtrup, Quantum Error Correction in a Solid-State Hybrid Spin Register, Nature (London) 506, 204 (2014).

[11] D. D. Awschalom, L. C. Bassett, A. S. Dzurak, E. L. Hu, and J.R. Petta, Quantum Spintronics: Engineering and Manipulating Atom-like Spins in Semiconductors, Science 339, 1174 (2013).

[12] R. Barends et al., Superconducting Quantum Circuits at the Surface Code Threshold for Fault Tolerance, Nature (London) 508, 500 (2014).

[13] J. Kelly et al., State Preservation by Repetitive Error Detection in a Superconducting Quantum Circuit, Nature (London) 519, 66 (2015).

[14] A. D. Córcoles, E. Magesan, S. J. Srinivasan, A. W. Cross, M. Steffen, J. M. Gambetta, and J. M. Chow, Demonstration of a Quantum Error Detection Code Using a Square Lattice of Four Superconducting Qubits, Nat. Commun. 6, 6979 (2015).

[15] A. Morello, J. J. Pla, F. A. Zwanenburg, K. W. Chan, K. Y. Tan, H. Huebl, M. Möttönen, C. D. Nugroho, C. Yang, J. A. van Donkelaar, A. D. C. Alves, D. N. Jamieson, C. C. Escott, L. C. L. Hollenberg, R. G. Clark, and A. S. Dzurak, Single-Shot Readout of an Electron Spin in Silicon, Nature (London) 467, 687 (2010).

[16] J. J. Pla, K. Y. Tan, J. P. Dehollain, W. H. Lim, J. J. L. Morton, D. N. Jamieson, A. S. Dzurak, and A. Morello, A Single-Atom Electron Spin Qubit in Silicon, Nature (London) 489, 541 (2012).

[17] J. J. Pla, K. Y. Tan, J. P. Dehollain, W. H. Lim, J. J. L. Morton, F. A. Zwanenburg, D. N. Jamieson, A. S. Dzurak, and A. Morello, High-Fidelity Readout and Control of a Nuclear Spin Qubit in Silicon, Nature (London) 496, 334 (2013).

[18] A. Ajoy, U. Bissbort, M. D. Lukin, R. L. Walsworth, and P. Cappellaro, Atomic-Scale Nuclear Spin Imaging Using Quantum-Assisted Sensors in Diamond, Phys. Rev. X 5, 011001 (2015).

[19] I. Lovchinsky, A. O. Sushkov, E. Urbach, N. P. de Leon, S. Choi, K. De Greve, R. Evans, R. Gertner, E. Bersin, C. Müller, L. McGuinness, F. Jelezko, R. L. Walsworth, H. Park, and M. D. Lukin, Nuclear Magnetic Resonance Detection and Spectroscopy of Single Proteins Using Quantum Logic, Science 351, 836 (2016).

[20] E. Togan, Y. Chu, A. S. Trifonov, L. Jiang, J. Maze, L. Childress, M. V. G. Dutt, A. S. Sørensen, P. R. Hemmer, A.S. Zibrov, and M.D. Lukin, Quantum Entanglement between an Optical Photon and a Solid-State Spin Qubit, Nature (London) 466, 730 (2010).

[21] H. Bernien, B. Hensen, W. Pfaff, G. Koolstra, M. S. Blok, L. Robledo, T. H. Taminiau, M. Markham, D. J. Twitchen, L. Childress, and R. Hanson, Heralded Entanglement between Solid-State Qubits Separated by Three Metres, Nature (London) 497, 86 (2013).

[22] B. Hensen, H. Bernien, A. E. Dréau, A. Reiserer, N. Kalb, M. S. Blok, J. Ruitenberg, R. F. L. Vermeulen, R. N. Schouten, C. Abellán, W. Amaya, V. Pruneri, M. W. Mitchell, M. Markham, D. J. Twitchen, D. Elkouss, S. Wehner, T. H. Taminiau, and R. Hanson, Loophole-Free Bell Inequality Violation Using Electron Spins Separated by 1.3 Kilometres, Nature (London) 526, 682 (2015).

[23] Kai-Mei C. Fu, C. Santori, P. E. Barclay, L. J. Rogers, N. B. Manson, and R. G. Beausoleil, Observation of the Dynamic Jahn-Teller Effect in the Excited States of Nitrogen-Vacancy Centers in Diamond, Phys. Rev. Lett. 103, 256404 (2009).

[24] P. Rabl, S. J. Kolkowitz, F. H. L. Koppens, J. G. E. Harris, P. Zoller, and M. D. Lukin, A Quantum Spin Transducer Based on Nanoelectromechanical Resonator Arrays, Nat. Phys. 6, 602 (2010).

[25] O. Arcizet, V. Jacques, A. Siria, P. Poncharal, P. Vincent, and S. Seidelin, A Single Nitrogen-Vacancy Defect Coupled to a Nanomechanical Oscillator, Nat. Phys. 7, 879 (2011).

[26] S. Kolkowitz, A. C. Bleszynski Jayich, Q. P. Unterreithmeier, S. D. Bennett, P. Rabl, J. G. E. Harris, and M. D. Lukin, Coherent Sensing of a Mechanical Resonator with a SingleSpin Qubit, Science 335, 1603 (2012).

[27] S. D. Bennett, N. Y. Yao, J. Otterbach, P. Zoller, P. Rabl, and M. D. Lukin, Phonon-Induced Spin-Spin Interactions in Diamond Nanostructures: Application to Spin Squeezing, Phys. Rev. Lett. 110, 156402 (2013).

[28] E. R. MacQuarrie, T. A. Gosavi, N. R. Jungwirth, S. A. Bhave, and G. D. Fuchs, Mechanical Spin Control of Nitrogen-Vacancy Centers in Diamond, Phys. Rev. Lett. 111, 227602 (2013).

[29] P. Ovartchaiyapong, K. W. Lee, B. A. Myers, and A. C. Bleszynski Jayich, Dynamic Strain-Mediated Coupling of a Single Diamond Spin to a Mechanical Resonator, Nat. Commun. 5, 4429 (2014).

[30] J. Teissier, A. Barfuss, P. Appel, E. Neu, and P. Maletinsky, Strain Coupling of a Nitrogen-Vacancy Center Spin to a Diamond Mechanical Oscillator Phys. Rev. Lett. 113, 020503 (2014).

[31] N. Y. Yao, L. Jiang, A. V. Gorshkov, P. C. Maurer, G. Giedke, J. I. Cirac, and M. D. Lukin, Scalable Architecture for a Room Temperature Solid-State Quantum Information Processor, Nat. Commun. 3, 800 (2012).

[32] P. Cappellaro, in Quantum State Transfer and Network Engineering, edited by G. M. Nikolopoulos and I. Jex (Springer, Heidelberg, 2014).

[33] O. D. Restrepo and W. Windl, Full First-Principles Theory of Spin Relaxation in Group-IV Materials, Phys. Rev. Lett. 109, 166604 (2012).

[34] Calculated using the predicted $T_{1} \sim 180 \mathrm{~ns}$ and the observed drift velocity $\sim 10^{6} \mathrm{~cm} / \mathrm{s}$ in high-purity diamond under the application of an electric field with magnitude $\sim 100 \mathrm{~V} / \mathrm{cm}$ [35]. 
[35] J. Isberg, M. Gabrysch, J. Hammersberg, S. Majdi, K. K. Kovi, and D. J. Twitchen, Generation, Transport and Detection of Valley-Polarized Electrons in Diamond, Nat. Mater. 12, 760 (2013).

[36] A. Jarmola, V. M. Acosta, K. Jensen, S. Chemerisov, and D. Budker, Temperature- and Magnetic-Field-Dependent Longitudinal Spin Relaxation in Nitrogen-Vacancy Ensembles in Diamond, Phys. Rev. Lett. 108, 197601 (2012).

[37] See, for example, B. Weber et al., Ohm's Law Survives to the Atomic Scale, Science 335, 64 (2012).

[38] L. C. L. Hollenberg, A. D. Greentree, A. G. Fowler, and C. J. Wellard, Two-Dimensional Architectures for DonorBased Quantum Computing, Phys. Rev. B 74, 045311 (2006).

[39] I. Žutić, J. Fabian, and S. Das Sarma, Spintronics: Fundamentals and Applications, Rev. Mod. Phys. 76, 323 (2004).

[40] I. Appelbaum, B. Huang, and D. J. Monsma, Electronic Measurement and Control of Spin Transport in Silicon, Nature (London) 447, 295 (2007).

[41] C. A. Meriles and M. W. Doherty, Generation of SpinPolarized Currents via Cross-Relaxation with Dynamically Pumped Paramagnetic Impurities, Appl. Phys. Lett. 105, 022403 (2014).

[42] N. Aslam, G. Waldherr, P. Neumann, F. Jelezko, and J. Wrachtrup, Photo-Induced Ionization Dynamics of the Nitrogen Vacancy Defect in Diamond Investigated by Single-Shot Charge State Detection, New J. Phys. 15, 013064 (2013).

[43] M. Nesládek, L. M. Stals, A. Stesmans, K. Iakoubovskij, G. J. Adriaenssens, J. Rosa, and M. Vaněček, Dominant Defect Levels in Diamond Thin Films: A Photocurrent and Electron Paramagnetic Resonance Study, Appl. Phys. Lett. 72, 3306 (1998).

[44] See, for example, S. Pezzagna, B. Naydenov, F. Jelezko, J. Wrachtrup, and J. Meijer, Creation Efficiency of NitrogenVacancy Centres in Diamond, New J. Phys. 12, 065017 (2010).

[45] M. L. Goldman, A. Sipahigil, M. W. Doherty, N. Y. Yao, S. D. Bennett, M. Markham, D. J. Twitchen, N. B. Manson, A. Kubanek, and M. D. Lukin, Phonon-Induced Population Dynamics and Intersystem Crossing in Nitrogen-Vacancy Centers, Phys. Rev. Lett. 114, 145502 (2015).

[46] M. L. Goldman, M. W. Doherty, A. Sipahigil, N. Y. Yao, S. D. Bennett, N. B. Manson, A. Kubanek, and M. D. Lukin, State-Selective Intersystem Crossing in Nitrogen-Vacancy Centers, Phys. Rev. B 91, 165201 (2015).

[47] P. Neumann, J. Beck, M. Steiner, F. Rempp, H. Fedder, P. R. Hemmer, J. Wrachtrup, and F. Jelezko, Single-Shot Readout of a Single Nuclear Spin, Science 329, 542 (2010).

[48] A. Dréau, P. Spinicelli, J. R. Maze, J.-F. Roch, and V. Jacques, Single-Shot Readout of Multiple Nuclear Spin Qubits in Diamond under Ambient Conditions, Phys. Rev. Lett. 110, 060502 (2013).

[49] R. G. Farrer, On the Subsitutional Nitrogen Donor in Diamond, Solid State Commun. 7, 685 (1969).

[50] F. J. Heremans, G. D. Fuchs, C. F. Wang, R. Hanson, and D. D. Awschalom, Generation and Transport of Photoexcited Electrons in Single-Crystal Diamond, Appl. Phys. Lett. 94, 152102 (2009).
[51] J. Isberg, A. Tajani, and D. J. Twitchen, Photoionization Measurement of Deep Defects in Single-Crystalline CVD Diamond Using the Transient-Current Technique, Phys. Rev. B 73, 245207 (2006).

[52] P. Deák, B. Aradi, M. Kaviani, T. Frauenheim, and A. Gali, Formation of NV Centers in Diamond: A Theoretical Study Based on Calculated Transitions and Migration of Nitrogen and Vacancy Related Defects, Phys. Rev. B 89, 075203 (2014).

[53] S. Han, in Diamond: Electronic Properties and Applications, edited by L. S. Pan and D. R. Kania (Springer Science, New York, 1995).

[54] A. Alkauskas, Q. Yan, and C. G. Van de Walle, FirstPrinciples Theory of Nonradiative Carrier Capture via Multiphonon Emission, Phys. Rev. B 90, 075202 (2014).

[55] W. Saslow, T. K. Bergstresser, and M. L. Cohen, Band Structure and Optical Properties of Diamond, Phys. Rev. Lett. 16, 354 (1966).

[56] M. S. Dresselhaus, G. Dresselhaus, and A. Jorio, Group Theory: Applications to the Physics of Condensed Matter (Springer, Berlin, 2008).

[57] J. H. N. Loubser and J. A. van Wyk, Electron Spin Resonance in the Study of Diamond, Rep. Prog. Phys. 41, 1201 (1978).

[58] A. M. Stoneham, Theoretical Status of Diamond and Its Defects, Excited States and Atomic Motion, Mater. Sci. Eng. B 11, 211 (1992).

[59] A. Cox, M. E. Newton, and J. M. Baker, ${ }^{13} \mathrm{C},{ }^{14} \mathrm{~N}$ and ${ }^{15} \mathrm{~N}$ ENDOR Measurements on the Single Substitutional Nitrogen Centre (P1) in Diamond, J. Phys. Condens. Matter 6, 551 (1994).

[60] J. H. N. Loubser and W. P. van Ryneveld, The Dynamic Jahn-Teller and Other Effects in the High-Temperature Electron Spin Resonance Spectrum of Nitrogen in Diamond, Br. J. Appl. Phys. 18, 1029 (1967).

[61] B. K. Ridley, Quantum Processes in Semiconductors, 5th ed. (Oxford University Press, Oxford, 2013).

[62] J. Isberg, J. Hammersberg, E. Johansson, T. Wikström, D. J. Twitchen, A. J. Whitehead, S. E. Coe, and G. A. Scarsbrook, High Carrier Mobility in Single-Crystal Plasma-Deposited Diamond, Science 297, 1670 (2002).

[63] Here, we make the simplifying approximation that the surface electrodes apply a uniform electric field $\vec{E}_{\text {ap }}=$ $-E \hat{z}$ in the region where transport is occurring. We assumed that $\vec{E}_{\text {ap }}$ is much larger than the monopole electric fields produced by the charged ionizing and capture centers, so that they can be ignored and $\vec{E} \approx \vec{E}_{\text {ap }}$. Since the capture rate $\sim 10 \mathrm{MHz}$ is much slower than the rates of ionization $\sim 0.1-1 \mathrm{GHz}$ and drift diffusion $>\mu \mathrm{m} / \mathrm{ns}$, it can be safely ignored when solving for the electron drift diffusion. As ionization and drift diffusion have similar rates, the initial condition we define to solve for the electron drift diffusion is not a true initial condition (as would be the case if ionization were much faster), but rather represents a time average of the electron probability density during ionization. This time average is quantified by the initial width $w$.

[64] Although this result is outside the applicable time scale for the drift-diffusion model, we still conclude that it implies that the simple architecture does not adequately confine the 
electron probability density to achieve the required capture rates.

[65] S. Seidelin, J. Chiaverini, R. Reichle, J. J. Bollinger, D. Leibfried, J. Britton, J. H. Wesenberg, R. B. Blakestad, R. J. Epstein, D. B. Hume, W. M. Itano, J. D. Jost, C. Langer, R. Ozeri, N. Shiga, and D. J. Wineland, Microfabricated Surface-Electrode Ion Trap for Scalable Quantum Information Processing, Phys. Rev. Lett. 96, 253003 (2006).

[66] L. C. Bassett, F. J. Heremans, C. G. Yale, B. B. Buckley, and D. D. Awschalom, Electrical Tuning of Single NitrogenVacancy Center Optical Transitions Enhanced by Photoinduced Fields, Phys. Rev. Lett. 107, 266403 (2011).

[67] T. M. Babinec, B. J. M. Hausmann, M. Khan, Y. Zhang, J. R. Maze, P. R. Hemmer, and M. Lončar, A Diamond Nanowire Single-Photon Source, Nat. Nanotechnol. 5, 195 (2010).

[68] P. Maletinsky, S. Hong, M. S. Grinolds, B. Hausmann, M. D. Lukin, R. L. Walsworth, M. Loncar, and A. Yacoby, A Robust Scanning Diamond Sensor for Nanoscale Imaging with Single Nitrogen-Vacancy Centres, Nat. Nanotechnol. 7, 320 (2012).

[69] S. A. Momenzadeh, R. J. Stöhr, F. F. de Oliveira, A. Brunner, A. Denisenko, S. Yang, F. Reinhard, and J. Wrachtrup, Nanoengineered Diamond Waveguide as a Robust Bright Platform for Nanomagnetometry Using Shallow Nitrogen Vacancy Centers, Nano Lett. 15, 165 (2015).

[70] P. Andrich, B. J. Alemán, J. C. Lee, K. Ohno, C. F. de las Casas, F. J. Heremans, E. L. Hu, and D. D. Awschalom, Engineered Micro- and Nanoscale Diamonds as Mobile Probes for High-Resolution Sensing in Fluid, Nano Lett. 14, 4959 (2014).

[71] A. Stacey, K. M. O’Donnell, J.-P. Chou, A. Schenk, A. Tadich, N. Dontschuk, J. Cervenka, C. Pakes, A. Gali, A. Hoffman, and S. Prawer, Nitrogen Terminated Diamond, Adv. Mater. 2, 1500079 (2015).

[72] A. W. Holleitner, V. Sih, R. C. Myers, A. C. Gossard, and D. D. Awschalom, Suppression of Spin Relaxation in Submicron InGaAs Wires, Phys. Rev. Lett. 97, 036805 (2006).

[73] J. Michl, T. Teraji, S. Zaiser, I. Jakobi, G. Waldherr, F. Dolde, P. Neumann, M. W. Doherty, N. B. Manson, J. Isoya, and J. Wrachtrup, Perfect Alignment and Preferential Orientation of Nitrogen-Vacancy Centers during Chemical Vapor Deposition Diamond Growth on (111) Surfaces, Appl. Phys. Lett. 104, 102407 (2014).

[74] H. J. Mamin, C. T. Rettner, M. H. Sherwood, L. Gao, and D. Rugar, High Field-Gradient Dysprosium Tips for Magnetic Resonance Force Microscopy, Appl. Phys. Lett. 100, 013102 (2012).

[75] V. Filidou, S. Simmons, S. D. Karlen, F. Giustino, H. L. Anderson, and J. J. L. Morton, Ultrafast Entangling Gates between Nuclear Spins Using Photoexcited Triplet States, Nat. Phys. 8, 596 (2012).

[76] To estimate $\mathcal{F}_{\mathrm{NV}}(\lambda)$, we first extract the $\mathrm{NV}^{-}$absorption spectrum from Ref. [77] and rescale it to units of absorption rate per unit optical power by comparing it to the partial photoexcitation spectrum in Ref. [42]. We then extract the one-photon photoionization spectrum from Ref. [42], which is in similar units of photoionization rate per unit optical power. We finally insert this data into the expression for $\mathcal{F}_{\mathrm{NV}}(\lambda)$. This estimation method allows rates in approximately the same optical conditions to be compared.

[77] G. Davies, Vibronic Spectra in Diamond, J. Phys. C 7, 3797 (1974).

[78] G. Waldherr, J. Beck, M. Steiner, P. Neumann, A. Gali, Th. Frauenheim, F. Jelezko, and J. Wrachtrup, Dark States of Single Nitrogen-Vacancy Centers in Diamond Unraveled by Single Shot NMR, Phys. Rev. Lett. 106, 157601 (2011).

[79] E. Bourgeois, A. Jarmola, P. Siyushev, M. Gulka, J. Hruby, F. Jelezko, D. Budker, and M. Nesládek, Photoelectrical Detection of Electron Spin Resonance of Nitrogen-Vacancy Centres in Diamond, Nat. Commun. 6, 8577 (2015).

[80] Conduction band degeneracy in diamond, and, therefore, spin-orbit mixing, first occurs $\sim 0.5 \mathrm{eV}$ above the conduction band minimum at the $X k$ point [55]. Owing to their spin-orbit mixing, photoionization to these $X_{1}$ Bloch spin orbitals will result in loss of spin coherence and polarization.

[81] The estimate of $66 \%$ probability is based on the simple picture that there is equal chance that any one of the three electrons occupying the $e$ spin orbitals in the ${ }^{3} E$ optical excited state are ejected. This picture is strictly true only if there is equal chance that the NV center's final state after photionization is any of the levels $\left({ }^{4} A_{2},{ }^{2} A_{2},{ }^{2} E,{ }^{2} A_{1}\right)$ of the $a_{1} e^{2}$ configuration. These levels are expected to be split by energies $(\sim 0.5 \mathrm{eV})$ comparable to optical energies. Thus, the final state of the NV center will depend on the optical excitation wavelength. We have worked through the implications of this complication and found that it does not alter our conclusions about the utility of the two-phonon mechanism.

[82] M. Kozák, F. Trojánek, B. Dzurňák, and P. Malý, Two- and Three-Photon Absorption in Chemical Vapor Deposition Diamond, J. Opt. Soc. Am. B 29, 1141 (2012).

[83] M. Katagiri, J. Isoya, S. Koizumi, and H. Kanda, Electron Paramagnetic Resonance Study of Phosphorus-Doped n-Type Homoepitaxial Diamond Films Grown by Chemical Vapor Deposition, Phys. Status Solidi A 203, 3367 (2006).

[84] M. Nesládek, K. Meykens, K. Haenen, L. M. Stals, T. Teraji, and S. Koizumi, Low-Temperature Spectroscopic Study of n-Type Diamond, Phys. Rev. B 59, 14852 (1999).

[85] B. Butorac and A. Mainwood, Symmetry of the Phosphorus Donor in Diamond from First Principles, Phys. Rev. B 78, 235204 (2008).

[86] S. Koizumi, M. Kamo, Y. Sato, S. Mita, A. Sawabe, A. Reznik, C. Uzan-Saguy, and R. Kalish, Growth and Characterization of Phosphorus Doped n-Type Diamond Thin Films, Diam. Relat. Mater. 7, 540 (1998).

[87] J. F. Prins, Ion-Implanted n-Type Diamond: Electrical Evidence, Diam. Relat. Mater. 4, 580 (1995).

[88] R. Jones, J. E. Lowther, and J. Goss, Limitations to n-Type Doping in Diamond: The Phosphorus-Vacancy Complex, Appl. Phys. Lett. 69, 2489 (1996). 